\title{
Role of the Mdm2 SNIP 309 Polymorphism in Gastric Mucosal Morphologic Patterns of Patients with Helicobacter pylori Associated Gastritis
}

\author{
Taweesak Tongtawee ${ }^{1,4 *}$ Chavaboon Dechsukhum $^{3,4}$, Wilairat Leeanansaksiri ${ }^{7}$, \\ Soraya Kaewpitoon ${ }^{2,4}$, Natthawut Kaewpitoon ${ }^{5,6}$, Ryan A Loyd ${ }^{2,4}$, Likit \\ Matrakool $^{1,4}$, Sukij Panpimanmas ${ }^{1,4}$
}

\begin{abstract}
Background: The tumor suppressor p53 is as a regulator of cell proliferation, apoptosis and many other biological processes as well as external and internal stress responses. Mdm2 SNIP309 is a negative regulator of 53. Therefore, this study aimed to determine the role of the Mdm2 SNIP 309 polymorphism in the gastric mucosal morphological patterns in patients with Helicobacter pylori associated gastritis. Materials and Methods: A prospective cross-sectional study was carried out from November 2014 through November 2015. Biopsy specimens were obtained from patients and infection was proven by positive histology. Gastric mucosa specimens were sent to the Molecular Genetics Unit, Institute of Medicine, Suranaree University of Technology where they were tested by molecular methods to detect the patterns of Mdm2 SNIP 309 polymorphism using the real-time PCR hybridization probe method. The results were analyzed and correlated with gastric mucosal morphological patterns by using C-NBI endoscopy. Results: A total of 300 infected patients were enrolled and gastric mucosa specimens were collected. In this study the percentage of Mdm2 SNIP 309 T/T homozygous and Mdm2 SNIP309 G/T heterozygous was 78\% and $19 \%$ respectively whereas Mdm2 SNIP309 G/G homozygous was 3\%. Mdm2 SNIP 309 T/T homozygous and Mdm2 SNIP309 G/T heterozygosity correlated with type 1 to type 3 gastric mucosal morphological patterns $(P<0.01)$ whereas Mdm2 SNIP309 G/G homozygous correlated with type 4 and type $5(P<0.01)$. Conclusions: Our study finds the frequency of Mdm2 SNIP309 G/G in a Thai population is very low, and suggests that this can explain ae Thailand enigma. Types 1 to type 3 are the most common gastric mucosal morphological patterns according to the unique genetic polymorphism of MDM2 SNIP 309 in the Thai population.
\end{abstract}

Keywords: MDM2 SNIP 309 - gastric mucosal morphologic patterns - H. pylori - C-NBI endoscopy

Asian Pac J Cancer Prev, 17 (3), 1057-1060

\section{Introduction}

Mdm2 was discovered in 1992 to bind to, and negatively regulate, transactivation of $\mathrm{p} 53$, and was then itself found to be a transcriptional target of p53, defining a negative feedback loop of p53 tumor suppressor gene (Momand et al.,1992; Picksley et al.,1993). Mdm2 proved to be an E3 ubiquitin ligase, stimulating p53 degradation (Haupt et al., 1997; Honda et al., 1997; Kubbutat et al., 1997). Besides the well-established role of MDM2 in carcinogenesis via the p53-dependent pathway, MDM2 has been shown to be involved in inflammatory process.

With regard to this pro-inflammatory property, MDM2 has p53-independent activation property for nuclear factor-kappa beta $(\mathrm{NF} x \mathrm{~B})$. Due the fact that p53 and nuclear factor-kappa beta $(\mathrm{NF} \varkappa \mathrm{B})$ are the reciprocal negative regulatory partners, inflammation and carcinogenesis are linked together. It has been shown that persistent activation of $\mathrm{NF} x \mathrm{~B}$ during chronic inflammation can lead to cancer development through inhibition of p53 function (Gurova et al., 2005; O'Prey et al., 2010). Moreover, p53 has anti-inflammatory activity via NFxB inhibition (Tergaonkar et al., 2002; Komarova et al., 2005). As MDM2 can regulate both p53 and NFxB, MDM2 altered expression likely contribute to both inflammation and carcinogenesis. According to this scenario, genetic polymorphism-mediated MDM2 overexpression likely disturbs the physiologic balance of p53 and NFxB in that pro-inflammatory and hyper-proliferative states are preferable.

${ }^{1}$ Department of Surgery, ${ }^{2}$ Family Medicine and Community Medicine, ${ }^{3}$ Pathological Unit, Institute of Medicine, ${ }^{5}$ Parasite Research Unit, ${ }^{7}$ Institute of Science, Suranaree University of Technology, ${ }^{4}$ Suranaree University of Technology Hospital, ${ }^{6}$ Faculty of Public Health, Vongchawalitkul University, Nakhonrachasima, Thailand*For correspondence: taweesak.t@sut.ac.th 
For this reason, we hypothesize that the specific genetic polymorphism of MDM2 gene (T309G gene polymorphism) with resulting MDM2 overexpression is also likely associated with more severe inflammation in $H$. pylori-associated gastritis.

The Narrow Band Imaging system (NBI) is an endoscopic imaging technique for enhanced visualization of mucosal microscopic structure and capillaries in the superficial mucosal layer. Images are obtained by using Narrow Band red, blue, and green filters, which are different from conventional red-green-blue filters (Gono et al., 2010). The appearance of $H$. pylori negative that was a normal stomach as well as alterations induced by $H$. pylori associated gastritis have been described (Yagi et al., 2002). Kyoto global consensus report on $H$.pylori gastritis suggests that atrophic mucosa and intestinal metaplasia can be accurately detected by image-enhanced endoscopy, after appropriate training (Kentaro et al., 2015).

According to our study for Conventional Narrow Band Imaging gastroscopy (C-NBI), the result shows good efficacy for diagnosis $H$. pylori infection and correlation to histopathology of gastric mucosa (Taweesak et al., 2015). We are interested in pursuing clinical study in the Thai population to evaluate the possible association between Mdm2 SNP309 and gastric mucosal morphologic patterns using C-NBI gastroscopy. As the inflammatory process is also influenced by p53, we also evaluated the possible association between this SNP and the degree of chronic gastritis associated with H.pylori, which is the precursor lesion for gastric cancer.

\section{Materials and Methods}

\section{Patients}

A total of 300 patients with histology diagnosed $H$. pylori associated gastritis had gastric tissue collected to participate in this study from November 2014 through November 2015. The study was performed in accordance with good clinical practice and the guidelines of the Declaration of Helsinki. All patients provided written informed consent and the study protocol was approved by the Ethics Committee for Research Involving Human Subjects, of Suranaree University of Technology (EC57-34).

\section{Biopsy specimens}

The gastroscopic procedures were performed using an upper GI video endoscope (Olympus EVIS EXERA III, CV-190). The whole stomach was examined first with conventional endoscopy. After the whole stomach mucosa was observed and then we chose site of specific gastric mucosa according to previous studies (Taweesak et al., 2015)

\section{Image evaluation}

All gastroscopic examinations were digitally recorded and still images of the observation sites were captured for the use in the reproducibility study. The selected images were transferred to a software program without distorting brightness, contrast or color balance. A total of 300 pictures from 300 patients were selected for the inter and intra observer agreement study. All endoscopists were blinded to the results of the H.pylori status and histology before reviewing the gastroscopic picture.

\section{Histological analysis}

Gastric tissue specimens were sent to the pathologist for histological analysis. The hematoxylin and eosin stain and the Giemsa stain were used for identification of $H$. pylori. The pathologica

1 analysis was made by 5 pathologists at Bangkok Pathological Laboratory outside of Suranaree University of Technology according to the Sydney classification (M. F. Dixon.,1996)(Table 1), including evaluation of chronic inflammation, atrophy, intestinal metaplasia, and activity of gastritis.

\section{DNA isolation method}

The DNA of H.pylori was extracted from frozen gastric tissue biopsy specimens which were stored at a temperature of less than $-20^{\circ} \mathrm{C}$ using the QIAamp DNA FFPE tissue kit (Qiagen, USA). The DNA extraction was performed according to the manufacturer protocol. Briefly, ten tissue sections of $5 \mu \mathrm{M}$ thick were collected in $1.5 \mathrm{ml}$ micro centrifuge tubes. The tissue specimens were placed in a microcentrifuge tube, and buffer ATL (180 $\mu \mathrm{L})$ and proteinase $\mathrm{K}(20 \mu \mathrm{L})$ were added. The samples were mixed by vortexing and incubated at $56^{\circ} \mathrm{C}$ until the tissues were completely lysed. Buffer AL $(200 \mu \mathrm{L})$ was added to the samples, which were subsequently incubated at $70^{\circ} \mathrm{C}$ for 10 minutes. Next, $240 \mu \mathrm{L}$ of $100 \%$ ethanol was added to the samples, which were mixed by vortexing for 15 seconds. Each sample was placed in a QIAamp spin column and centrifuged at $8000 \mathrm{rpm}$ for 1 minute. The

Table 2. Patient Demographic Data

\begin{tabular}{lc}
\hline Demographics data & $\mathrm{n}=300$ \\
\hline Male/female (n) & $72 / 228$ \\
Mean age (years) & 48.2 \\
Peptic ulcer disease (GU/DU) & $15 \%$ \\
Gastric cancer & $1 \%$ \\
Non ulcer gastritis/duodinitis & $84 \%$ \\
\hline
\end{tabular}

Table 1. Updated Sydney System

\begin{tabular}{|c|c|c|c|c|}
\hline Histologic properties & Definition & mild & Grademoderate & severe \\
\hline Chronic inflammation & Lymphocyte and plasma cell in lamina propria & $1+$ & $2+$ & $3+$ \\
\hline Neutrophil activation & Neutrophilic infiltration in lamina propria or superficial epithelium & $<1 / 3$ & $1 / 3-2 / 3$ & $>2 / 3$ \\
\hline Glandular atrophy & Loss of corpus and antral glands & $1+$ & $2+$ & $3+$ \\
\hline Intestinal metaplasia & Intestinal metaplasia of mucosal epithelium & $3+$ & $1 / 3-2 / 3$ & $>2 / 3$ \\
\hline Helicobacter pylori & Helicobacter pylori intensity & $1+$ & $2+$ & $3+$ \\
\hline
\end{tabular}


Role of Mdm2 SNIP 309 Polymorphism in Gastric Mucosal Morphologic Patterns of Patients with H.pylori Associated Gastritis

Table 3. Patterns of Mdm2 SNIP 309 and the Gastric Mucosal Morphologic Patterns

\begin{tabular}{cccccc}
\hline Patterns of & \multicolumn{5}{c}{ Gastric Mucosal Morphologic Patterns } \\
\cline { 2 - 6 } $\begin{array}{c}\text { Mdm2 SNIP } \\
309\end{array}$ & Type 1 & Type 2 & Type 3 & Type 4 & Type 5 \\
\hline MDM2 & & & & & \\
$\begin{array}{c}\text { SNP309 T/T } \\
\text { homozygous }\end{array}$ & 147 & 39 & 21 & - & - \\
MDM2 & & & & & \\
$\begin{array}{c}\text { SNP309 G/T } \\
\text { heterozygous } \\
\text { MDM2 }\end{array}$ & 24 & 27 & 6 & - & - \\
$\begin{array}{c}\text { SNP309 G/G } \\
\text { homozygous }\end{array}$ & - & - & - & - & 6 \\
\hline
\end{tabular}

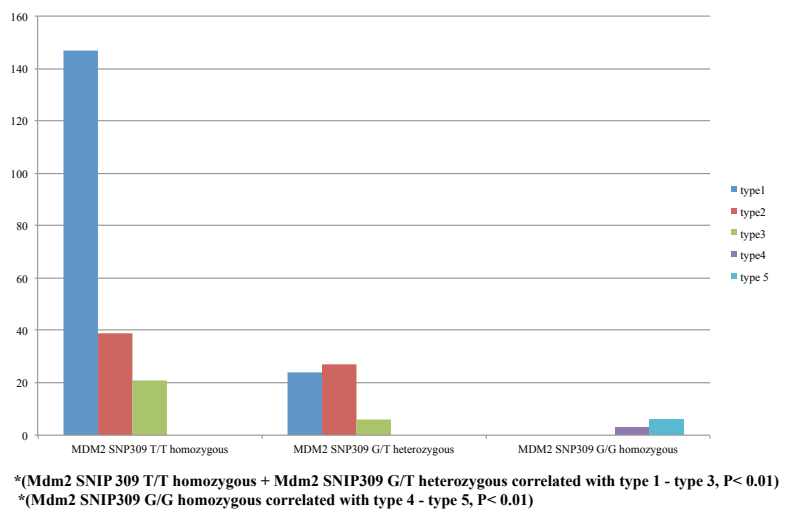

Figure 1. Pattern of MDM2 SNIP 309 and Gastric Mucosal Morphologic Patterns

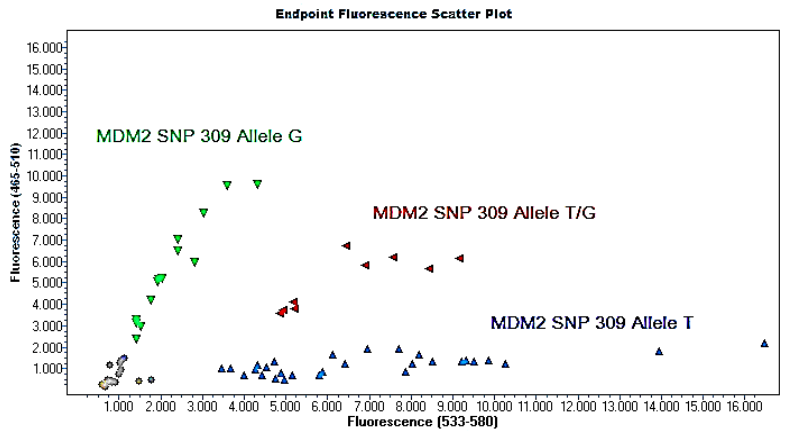

Figure 2. Pattern of genetic MDM2 SNIP 309 polymorphism using real-time PCR hybridization probes (light-cycler probe)

columns were washed with AW1 buffer $(500 \mu \mathrm{L})$, and samples were centrifuged at $8000 \mathrm{rpm}$ for 1 minute. AW2 buffer $(500 \mu \mathrm{L})$ was added to the column, and samples were centrifuged at 14,000 rpm for 3 minutes. Buffer AE $(200 \mu \mathrm{L})$ was added to each sample, and samples were incubated for 1 minute prior to centrifugation at 8000 rpm for 1 minute. Finally, the DNA was extracted from the tissue.

\section{Polymorphism analysis}

MDM2 SNP309 genotypes were analyzed using real-time PCR. The hybridization probes (light-cycler probe) were utilized for this analysis. The real-time PCR procedure was accomplished by using a LightCycler ${ }^{\circledR}$ 480 instrument (Roche diagnostics, Neuilly sur Seine,
France). The identifications of target PCR products were accomplished by melting curve analyses. The target PCR products were amplified by using the primers as reported in the previous literature. The hybridization probes included the one that was in the SNP309 (the sensor probe). This sensor probe is labeled by LC-red 640 at 5 ' and phosphorylated at 3 '. The anchor probe was hybridized to the PCR product at the site $3 \mathrm{bp}$ upstream from the sensor probe. $3 \mu 1$ of DNA templates were subjected to PCR reaction in the final volume of $20 \mu 1$. The reaction mixture consisted of $\mathrm{MgCl} 2(25 \mathrm{mM})$, forward and reverse primers (20 M each), sensor and anchor probes (20 M each), and 2 $\mu 1$ of FastStart DNA Master Hybridization Probes (Roche Diagnostics). PCR amplification comprised an initial denaturation cycle at $95^{\circ} \mathrm{C}$ for $10 \mathrm{~min}$, followed by 50 amplification cycles (with a temperature transition rate of $20^{\circ} \mathrm{C} / \mathrm{s}$ ) consisting of $95^{\circ} \mathrm{C}$ for $0 \mathrm{~s}$, annealing at $60^{\circ} \mathrm{C}$ for $10 \mathrm{~s}$, and extension at $72^{\circ} \mathrm{C}$ for $17 \mathrm{~s}$. After amplification a melting step was performed, consisting of $95^{\circ} \mathrm{C}$ for 0 $\mathrm{s}$, cooling to $45^{\circ} \mathrm{C}$ for $30 \mathrm{~s}$ (with a temperature transition rate of $20^{\circ} \mathrm{C} / \mathrm{s}$ ), and finally a slow rise in the temperature to $85^{\circ} \mathrm{C}$ at a rate of $0.1^{\circ} \mathrm{C} / \mathrm{s}$ with continuous acquisition of fluorescence decline. The genotype of each patient can be categorized into three genotypes SNP309 G/G homozygous, T/T homozygous and G/T heterozygous based on the different melting curves.

\section{Statistical analysis}

SPSS for Windows (version 16.0; SPSS, Chicago, IL, USA) was used for the statistical analysis; baseline demographic data as well as the correlation between pattern of Mdm2 SNIP 309 and the Gastric Mucosal Morphologic Patterns of the infected patients were analyzed. The patterns of genetic polymorphism were analyzed by using LightCycler ${ }^{\circledR} 480$ instrument (Roche diagnostics, Neuilly sur Seine, France).

\section{Results}

A total of 300 patients with histology diagnosed H.pylori associated gastritis had gastric tissue collected (72 men, 228 women; mean age 48.2 years, range 19-69 years). The patients' demographic data are summarized in Table 2. Patterns of Mdm2 SNIP 309 and the Gastric Mucosal Morphologic Patterns are summarized in Table 3. Mdm2 SNIP 309 T/T homozygous and Mdm2 SNIP309 G/T heterozygous was $78 \%$ and $19 \%$ respectively whereas Mdm2 SNIP309 G/G homozygous was 3\%. Mdm2 SNIP 309 T/T homozygous and Mdm2 SNIP309 G/T heterozygous correlated with type 1 to type 3 Gastric Mucosal Morphologic Patterns $(\mathrm{P}<0.01)$ whereas Mdm2 SNIP309 G/G homozygous correlated with type 4 and type 5 Gastric Mucosal Morphologic Patterns $(\mathrm{P}<0.01)$ (Figure 1). The pattern of the Mdm2 SNIP 309 genetic polymorphism using real-time PCR hybridization probes (light-cycler probe) is shown in Figure 2. Table 4 show the Inter and intraobserver agreement.

\section{Discussion}

H. pylori infection associated gastritis and gastric 
Taweesak Tongtawee et al

cancer risk remains a major health problem many countries in Asia such as China, Japan except Thailand, the term is "Thailand enigma". Cancer occurs through damage to the cell cycle, especially the tumor suppressor p53 as well as Mdm2 SNIP309, the negative regulator of p53. This study is the first to report the percentage of Mdm2 SNIP 309 T/T homozygous, Mdm2 SNIP309 G/T heterozygous, and Mdm2 SNIP309 G/G homozygous and correlation them with Gastric Mucosal Morphologic Patterns by using C-NBI endoscopy in Thai population. A previous case-control study among the Iranian gastric cancer population showed that Mdm2 SNP309 is a risk factor for this cancer (Moradi et al., 2014). Moreover, a study in a Chinese gastric cancer population also showed the same trend (Wang, 2009). Kyoto global consensus report on $H$. pylori gastritis suggests that atrophic mucosa and intestinal metaplasia can be accurately detected by image-enhanced endoscopy, after appropriate training (Kentaro et al., 2015). From the study, the result shows that. H.pylori infected gastric mucosa can be reliably identified using C-NBI gastroscopy and can also predict the histopathological severity of gastritis (Taweesak et al., 2015). In addition, Mdm2 SNIP 309 T/T homozygosity and Mdm2 SNIP309 G/T heterozygosity were correlated with type 1 to type 3 which represent of mild to moderate inflammation whereas Mdm2 SNIP309 G/G homozygousity was correlated with type 4 and type 5 which represent of severe inflammation. This study suggests that there is a correlation between patterns of Mdm2 SNP309 with gastric cancer development.

This study has demonstrated the good correlation of MDM2 SNP309 genotype and severity of inflammation in H. pylori infected gastric mucosa according to Gastric Mucosal Morphologic Patterns using C-NBI gastroscopy. The GG genotype of SNP309 enhanced the expression of MDM2 RNA and protein (Bond et al., 2005). The severity of inflammation may be contributed from the high expression of MDM2 protein in MDM2 SNP309 GG genotype.

In conclusion: Our study suggests that the frequency of Mdm2 SNP309 G/G is very low among Thai people, which can explain low gastric cancer changes in the Thai population. Good correlation between patterns of MDM2 SNIP 309 and Gastric Mucosal Morphologic Patterns using C-NBI gastroscopy.

\section{Acknowledgements}

This study was supported by a grant for medical investigation from Suranaree University of Technology and was approved by the Ethics Committee for Research Involving Human Subjects, Suranaree University of Technology (EC-57-34)

\section{References}

Bond GL, Hu W, Levine AJ, et al (2005). MDM2 is a central node in the $\mathrm{p} 53$ pathway: 12 years and counting. Curr Cancer Drug Targets, 5, 3-8.

Gono K, Obi T, Yamaguchi M, et al (2004). Appearance of enhanced tissue features in narrow-band endoscopic imaging. J Biomed Opt, 9, 568-77.

Gurova KV, Hill JE, Guo C, et al (2005). Small molecules that reactivate p53 in renal cell carcinoma reveals a nf-kappabdependent mechanism of p53 suppression in tumors. Proc Natl Acad Sci USA., 102, 17448-53.

Haupt Y, Maya R, et al (1997). Mdm2 promotes the rapid degradation of p53. Nature, 387, 296- 9.

Honda R, Tanaka H, et al (1997). Oncoprotein MDM2 is an ubiquitin ligase $\mathrm{E} 3$ for tumor suppressor p53. FEBS Letters, 420, 25-7.

Kentaro S, Jan T, Ernst JK, et al (2015). Kyoto global consensus report on Helicobacter pylori gastritis. Gut, 64, 1353- 67.

Komarova EA, Krivokrysenko V, Wang K, et al (2005). P53 is a suppressor of inflammatory response in mice. FASEB $J$, 19, 1030- 2 .

Kubbutat MH, Jones SN, et al (1997). Regulation of p53 stability by Mdm2. Nature, 387, 299-303.

MF Dixon, RM Genta JH, Harley et al (1996). "Classification and grading of gastritis. The Updated Sydney system. Am J Surgical Pathol, 20, 1161-81.

Momand J, Zambetti GP, et al (1992). The mdm-2 oncogene product forms a complex with the $\mathrm{p} 53$ protein and inhibits p53-mediated transactivation. Cell, 69, 1237- 45.

Moradi MT, Salehi Z, Aminian K, et al (2014). Effects of p53 codon 72 and MDM2 SNP309 polymorphisms on gastric cancer risk among the Iranian population. Asian Pac J Cancer Prev, 15, 7413-7.

O'Prey J, Crighton D, Martin AG, et al (2010). P53-mediated induction of noxa and p53aip1 requires nfkappab. Cell Cycle, 9, 947-52.

Picksley SM,Lane DPet al (1993).The p53-mdm2 autoregulatory feedback loop: a paradigm for the regulation of growth control by p53? Bioessays, 15, 689-90.

Taweesak T, Soraya K, Natthawut K, et al (2015). Correlation between gastric mucosal morphologic patterns and histopathological severity of helicobacter pylori associated gastritis using conventional narrow band imaging gastroscopy. Bio Med Res Int, 7, 42-8.

Tergaonkar V, Pando M, Vafa O, et al (2002). P53 stabilization is decreased upon nf kappa b activation: A role for nfkappab in acquisition of resistance to chemotherapy. Cancer Cell, 1, 493-503.

Wang X, Yang J, Ho B, et al (2009). Interaction of Helicobacter pylori with genetic variants in the MDM2 promoter is associated with gastric cancer susceptibility in Chinese patients. Helicobacter, 14, 114-9.

Yagi K, Nakamura A, Sekine A, et al (2002). Comparison between magnifying endoscopy and histological, culture and urease test findings from the gastric mucosa of the corpus. Endoscopy, 34, 376-81. 